\title{
Probability Model Deduction Method for Geotechnical Parameters Based on Normal Information Diffusion Principle
}

\author{
Shang-Qian Hou, Feng-Qiang Gong
}

School of Resources and Safety Engineering, Central South University, Changsha 410083, China

\begin{abstract}
Keywords: normal information diffusion; geotechnical parameter; probability model
Abstract: In order to reflect the randomness and uncertainty of geomaterials, it is essential to study the probability model of geotechnical parameters. Based on normal information diffusion principle, a probability model deduction method for geotechnical parameters was presented in this paper. Taken a set of friction coefficient data as basic information, deducted the probability distribution using normal information diffusion method. Then, K-S test was used to compare the results of classical distribution, truncated distribution and normal information diffusion distribution. The results show that, the test value of normal information diffusion distribution is smaller than the classical distribution and truncated distribution. The cumulative probability value of normal information diffusion distribution is 1 , and the cumulative probability curve of which is closer to the empirical cumulative distribution function. It turns out to be a better probability model deduction method for geotechnical parameters.
\end{abstract}

\section{Introduction}

With the development of geotechnical engineering theory, uncertainty analysis is widely adopted and used gradually. It's essential and significant to deduct the probability distributions of random variables for reliability analysis and optimized analysis.

H.S. Ang [1] has made outstanding contributions to the civil engineering reliability analysis. In his book, some important probability concepts and reliability methods are introduced. Traditional method for deducting the probability distribution of geotechnical parameters is using some classical distributions and goodness-of-fit tests. In the research of Chun-Ling Yan et al. [2], 50 compressive and 50 tensile strength values were obtained by RMT-150B testing systems. By the hypothesis test method, it was proved that the uniaxial compressive strength followed normal distribution, the tensile strength followed lognormal distribution, the elastic modulus and Poisson's ratio followed normal distribution, the cohesion followed lognormal distribution and the internal friction angle followed normal distribution. However, classical distributions cannot reflect the actual regularities of geotechnical parameters in many cases. Feng-Qiang Gong et al. [3] used Chebyshev orthogonal polynomial to deduct the probability distributions of rock random variables and proved this method was a better way. But, sometimes, this method can lead to the probability value being negative.

The principle of normal information diffusion (NID) based on fuzzy set methods is a new approach for functional approximation. The NID method can make sure the raw information is retained furthest [4]. Feng-Qiang Gong et al. [5] proved using this method to deduct the probability distributions of $c$ and $\varphi$ values was great. However, the truncation processing was not considered in their research. To verify the applicability of deducting probability distribution of geotechnical parameters using NID method, a set of friction coefficient sample will be taken as an example in this paper. This sample was considered to follow lognormal distribution [6]. Then, NID method is used to deduct the probability model and compared with classical distribution. In this way, it can be concluded whether NID distribution reflects the actual regularities of geotechnical parameters better.

\section{Probability Model Deduction Based on NID Principle}

The principle of normal information diffusion based on fuzzy set methods is a new approach for functional approximation. This method can transit raw information to fuzzy relation directly in a certain way. In this way, the calculation about membership function can be avoided. So the raw information can be retained furthest [4]. 


\section{Normal Information Diffusion}

Suppose a function $f(x)$ as the probability density function of random variable $O$. Define $\mu(x)$ as a Borel measurable function on $(-\infty, \infty) . X$ is a sample from population $O$. $W=\left\{w_{1}, w_{2}, \mathrm{~L}, w_{n}\right\}$ is the knowledge sample. $V$ is the domain of discourse of $W$ and $x_{i}$ is the observed value of $w_{i}$. Suppose $\Delta_{n}>0$ is the window width and it is a constant. Then, based on the principle of normal information diffusion, the estimation of distribution of $f(x)$ is

$$
\tilde{f}_{n}(x)=\frac{1}{\sqrt{2 \pi} n h} \sum_{i=1}^{n}\left\{\exp \left[-\frac{\left(x-x_{i}\right)^{2}}{2 h^{2}}\right]\right\} .
$$

where $h$ is the window width of standard normal diffusion $\mu(x)$ and $n$ is the number of samples.

Normal diffusion model is the most commonly used model of one-dimension information diffusion, which has been described and expressed by Huang [4].

The observed value of $w_{i}$ is $x_{i}$, the maximum of $x_{i}$ is $x_{\max }$, the minimum of $x_{i}$ is $x_{\min }$. According to the principle of selecting near in NID theory, the window width $h$ can be obtained as follows:

$$
h=\gamma\left(x_{\max }-x_{\min }\right) /(n-1) .
$$

The values of $\gamma$ can be got in a table [7].

\section{Fundamentals of Truncated Distribution}

In reliability design of geotechnical engineering, theoretical distributions are always used to deduct the probability model of random variables to make calculation easy, such as stress, strength, load, geometric dimensioning and so on. The value ranges of these random variables in theoretical distributions are all $(-\infty, \infty)$, which are improper in geotechnical engineering. Actually, the values of geotechnical parameters cannot be infinity, zero or negative infinity. So, the truncation processing is required, which will lead to the cumulative probability in truncation interval being less than 1 for theoretical distributions. Although the truncated distribution can make sure that the cumulative probability is 1 , this method is a linear processing ultimately without any practical significance.

The truncated distribution means that the original probability density function $f(x)$ divides a normalized constant $K$.

$$
K=\int_{a}^{b} f(x) d x .
$$

where $a, b$ are two truncation points respectively. If only one end is truncated, the other end is set to infinity or negative infinity.

The model of truncated distribution is as follows:

$$
F(t)=\left\{\begin{array}{l}
1, t<t_{\text {min }} \\
\int_{t}^{t_{\max }} f_{T}^{*}(t) d t, t_{\text {min }}<t<t_{\text {max }} . \\
0, t>t_{\max }
\end{array}\right.
$$

$f_{T}^{*}(t)$ is the truncated probability density function of $T$.

$f_{T}^{*}(t)=f_{T}(t) / K$.

$f_{T}(t)$ is the probability density function of theoretical distribution corresponding to the truncated distribution. 


$$
K=\int_{t_{\min }}^{t_{\max }} f_{T}(t) d t
$$

\section{Engineering Example}

Take a set of shale friction coefficient data (shown in Table 1) as the example. Xi-Bing Li et al. [6] considered that this sample followed log-normal distribution by traditional method. NID method deduction will be made and compared with the former.

Table 1 Data of shale friction coefficient [6].

\begin{tabular}{lccccccc}
\hline 0.88 & 0.83 & 0.94 & 0.74 & 0.97 & 0.92 & 0.9 & 0.71 \\
0.74 & 0.65 & 0.76 & 0.72 & 0.77 & 0.74 & 0.68 & 0.72 \\
0.78 & 0.35 & 0.3 & 0.46 & 0.44 & 0.9 & 0.27 & 0.48 \\
0.55 & 0.34 & 0.58 & 0.66 & 0.73 & & & \\
\hline
\end{tabular}

Fig. 1 shows that the probability density curve of NID distribution agrees with the actual distribution pretty well, from which the great superiority of this method can be seen intuitively. The cumulative probability curves also show the great superiority of NID method.

The K-S test results are listed in Table 2 and show that all of these distributions can pass the test. However, great superiority of NID distribution can be found by comparing the test results and the values of $D_{i}(n)$.
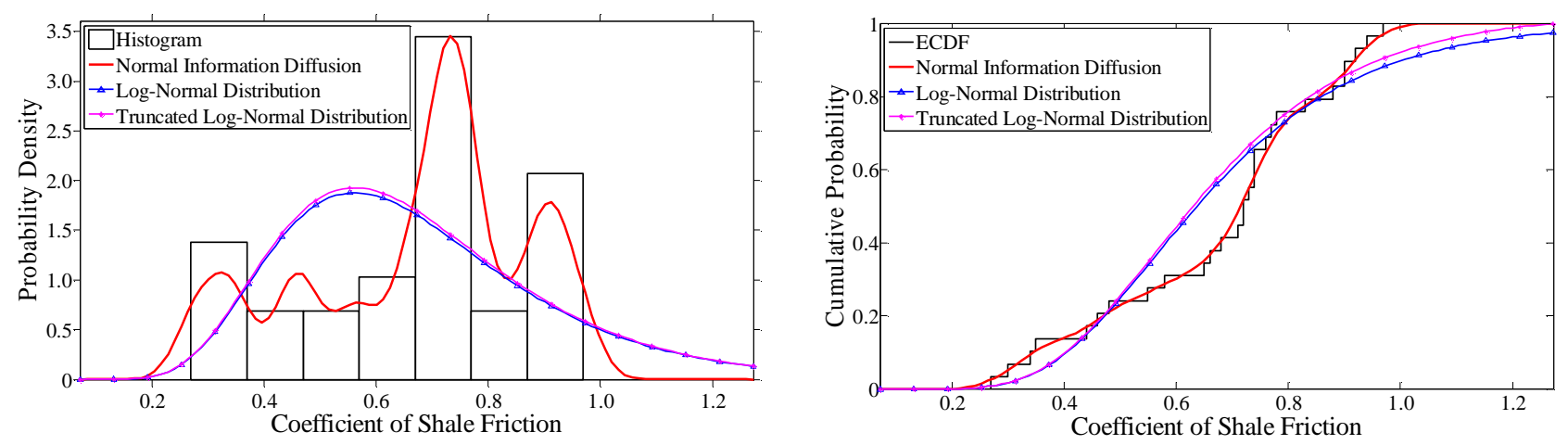

Fig. 1 Probability density and cumulative probability curves of shale friction coefficient.

Table 2 K-S test results of probability density function of shale friction coefficient.

\begin{tabular}{cccccc}
\hline \multirow{2}{*}{$\begin{array}{c}\text { Sample } \\
\text { Interval }\end{array}$} & Number & $\begin{array}{c}\text { Actual } \\
\text { Frequency }\end{array}$ & NID & Log-normal & $\begin{array}{c}\text { Truncated } \\
\text { Log-normal }\end{array}$ \\
\cline { 4 - 6 }$(0,0.31]$ & 2 & 0.0690 & 0.0036 & 0.0465 & 0.0459 \\
$(0,0.55]$ & 8 & 0.2759 & 0.0092 & 0.0678 & 0.0769 \\
$(0,0.79]$ & 22 & 0.7586 & 0.0327 & 0.0284 & 0.0091 \\
$(0,1.03]$ & 29 & 1.0000 & 0.0016 & 0.0872 & 0.0630 \\
$(0,1.27]$ & 29 & 1.0000 & 0.0000 & 0.0258 & 0.0000 \\
$D(\mathrm{n})$ & & 0.0327 & 0.0872 & 0.0769 \\
\hline$F$ & & \multicolumn{5}{c}{0.2400} \\
\hline Critical Value & & \multicolumn{5}{c}{0.9742} \\
\hline
\end{tabular}

For the sample of shale friction coefficient, the critical value is 0.2400 . The test results of log-normal distribution, truncated log-normal distribution and normal information diffusion distribution are 0.0872, 0.0769 and 0.0327. The NID distribution is the best method judging from the $\mathrm{K}-\mathrm{S}$ test. In addition, it can be found that the truncation processing rarely works for improving imitative effect.

The cumulative probability value of log-normal distribution is 0.9742 . The cumulative probability 
values of truncated log-normal distribution and NID distribution are both 1 . What's more, although the cumulative probability values of truncated log-normal distribution are 1, the probability density curve is unsatisfactory. Therefore, this linear processing is not an appropriate improved way.

\section{Conclusions}

By analyzing the above example, it can be seen that the K-S test results of NID distribution are better than the other two methods. The cumulative probability value of NID distribution can reach 1 . So, it can be concluded that using NID method to deduct the probability model of geotechnical parameters is appropriate. In this way, the probability model of geotechnical parameters can be more accurate.

\section{Acknowledgements}

This work was financially supported by the National Natural Science Foundation of China (41102170) and Independent Innovation Program Foundation of Central South University (2014zzts271).

\section{References}

[1] H.S. Ang and W.H. Tang: Probability concepts in engineering: emphasis on applications to civil and environmental engineering, John Wiley and Sons Publising, New York (2007), in press.

[2] C.L. Yan, D.X. Ding, Y.Q. Tang and Z.W. Bi: Testing of strength parameters and deformation parameters of surrounding rock and their distributions, Advanced Materials Research Vol. 261-263 (2011), p. 1360-1364.

[3] F.Q. Gong, S.Q. Hou and T.Y. Wu: Probability function estimation of rock random variables using Chebyshev orthogonal polynomial, Applied Mechanics \& Materials, Vol. 351-352 (2013), p. 1673-1676.

[4] C.F. Huang and Y. Shi: Towards Efficient Fuzzy Information Processing, Springer-Verlag Publising, Berlin Heidelberg GmbH (2002).

[5] F.Q. Gong, S.Q. Hou and X.M. Yan: Probability model deduction method of Mohr-Coulomb criteria parameters based on normal information diffusion principle, Chinese Journal of Rock Mechanics and Engineering, Vol. 32 (2013), p. 2225-2234.

[6] X.B. Li and F.Q. Gong: A method for fitting probability distributions to engineering properties of rock masses using Legendre orthogonal polynomials, Structural Safety Vol. 31 (2009), p. 335-343.

[7] X.Z. Wang: The theory, method and robustness of the parameter estimation based on the principle of information spread, Journal of Wuhan Technical University of Surveying and Mapping Vol. 24 (1999), p. 240-244. 